\title{
Ontology Matching: An Ultimate Solution for Semantic Interoperability in Healthcare
}

\author{
Olaronke Iroju \\ Adeyemi College of Education \\ Computer Science Department, \\ Ondo State, Nigeria
}

\author{
Abimbola Soriyan \\ Obafemi Awolowo University \\ Computer Science \& \\ Engineering Department
}

\author{
Ishaya Gambo \\ Obafemi Awolowo University \\ Computer Science \& \\ Engineering Department, lle-Ife
}

\begin{abstract}
The healthcare domain is a complex domain which lacks a unified terminological set, most especially in clinical cases. As a result of this, the messaging standards employed in the healthcare domain use different terms for the same concept which often results in clinical misinterpretation, knowledge mismanagement, misdiagnosis of the patient's illness or even death. Consequently, the healthcare system is characterized by high error rate and semantic heterogeneity. A lot of efforts have been made to resolve this problem through the use of standards, clinical terminologies, web services as well as the use of achetype. However, these solutions have proved unsuccessful in resolving semantic heterogeneity in healthcare. Ontologies have also been developed to resolve this problem by making explicit the meaning of terms used in healthcare. Ontologies provide a source of shared and precisely defined terms, resulting in interoperability by knowledge sharing and reuse. Unfortunately, the variety of ways that the healthcare domain is conceptualized results in the creation of different ontologies with contradicting or overlapping parts. Thus, the available ontologies also introduce semantic heterogeneity to this domain. An effective solution to this problem is the introduction of methods for finding matches among the various components of ontologies in healthcare in order to facilitate semantic interoperability. Therefore, this paper aims at examining the various attempts for achieving semantic interoperability in healthcare and also motivates the critical needs for ontology matching in healthcare systems.
\end{abstract}

\section{General Terms}

Ontology, Interoperability, Semantic.

\section{Keywords}

Ontology, ontology matching, semantic heterogeneity, interoperability, semantic interoperability.

\section{INTRODUCTION}

The healthcare domain is a complex domain that requires a consensus on the terms used amongst communicating systems in order to meet the information needs of patients as well as healthcare providers. However, this need is hampered by the fact that the healthcare domain lacks a unified terminological set. Consequently, the messaging or communicating standards employed in this domain use different terminologies for the same concept (e.g. heart and cardiac) which often results in severe semantic interoperability problem. Semantic interoperability is defined as the ability of information shared by systems to be understood at the level of formally defined domain concepts so that the information is machine processeable by the receiving system [1]. This is with the aim of increasing patient safety, reducing medical errors, improving efficiency as well as reducing medical costs. However, to realize these benefits, there is a need to address interoperability in healthcare at the semantic level.

Ontologies has been seen as a major solution to semantic interoperability because it describes the semantics of information sources and makes its contents explicit by providing a shared comprehension of a given domain of interest [2]. There are different kinds of ontologies employed in healthcare; these include Logical Observation Identifier Names Code (LOINC), OpenGalen, Medical Subject Heading (Mesh) and Systemized Nomenclature of Medicine-Clinical Terms (SNOMED-CT). Unfortunately, these ontologies are unfamiliar and unnatural to most healthcare providers [1]. Also, the use of diverse ontologies in the healthcare domain raises heterogeneity problems to a higher level. Hence, the healthcare domain is not semantically interoperable [3]. This problem can be resolved by matching the diverse ontologies [4]. Ontology matching finds correspondences between semantically related entities of the ontologies in order to reduce heterogeneity between them. Ontology matching enables the knowledge and data expressed in the matched ontologies to interoperate.

This paper therefore presents the concepts of interoperability as well as the different ways that have been employed to resolve the problem of semantic interoperability in healthcare. The paper also presents the concepts of ontology and ontology matching as well as its relevance to the healthcare domain.

\section{INTEROPERABILITY IN}

\section{HEALTHCARE}

Interoperability according to [5] is the ability of Information and Communication Technology (ICT) systems and of the business processes they support to exchange data and to enable the sharing of information and knowledge. The IEEE association according to [6] also viewed interoperability as the ability of two or more systems or components to exchange information and to use the information that has been exchanged. Interoperability in the Health system is the ability facilitated by ICT applications and systems to exchange, understand and act on citizens/patients and other health-related information and knowledge among linguistically and culturally disparate health professionals, patients and other actors and organizations within and across health system jurisdictions in a collaborative manner [2]. There are two basic levels of interoperability that are required for seamless exchange of information and understanding of information in the healthcare domain. These include technical or syntactic interoperability and semantic interoperability. Syntactic interoperability is referred to as the ability of 
systems to exchange data and services using a common interoperability protocol such as the High Level Architecture (HLA), while semantic interoperability is the ability of systems to exchange data with an unambiguous shared meaning [7]. The goal of semantic interoperability is to ensure that systems are able to recognize and process semantically equivalent information homogeneously, even if instances are heterogeneously represented, i.e. if they are differently structured, and/or using different terminology or different natural language [2]. Semantic interoperability is the most significant to healthcare systems according to [2]. This is because it involves the provision of high quality healthcare services at a reasonable cost, it ensures safe and meaningful sharing of health records between heterogeneous systems and healthcare providers and it also ensures consistent clinical care and information standards. Semantic interoperability also ensures data quality and consistency which enables meaningful and reliable use of longitudinal and heterogeneous data for public health, research and health service management [8]. In spite of all these numerous benefits of semantic interoperability to the healthcare domain, semantic interoperability of information in the healthcare sector is yet to be achieved [19] and [10]. However, there are different attempts that have been made to achieve semantic interoperability in healthcare to no avail. Some of these include.

\subsection{Standardization}

One of the major goals of the healthcare industry is to facilitate the exchange of patient and other health related information among diverse systems in the consortium of care. However, in order to realize this objective, healthcare systems have to adhere to the same standard. However, healthcare institutes do not conform to a single standard. For instance, the titles and codes of case reports, drugs, diseases, and examination vary in different hospitals. The definition, description and practice operation in the healthcare domain are also different. Thus, for systems in this domain to be totally interoperable, there must be unified and authoritative hospital standardization data dictionaries. The importance of conforming to a standard in the healthcare domain are to improve patient care by allowing interoperability among disparate systems [11] as shown in figure 1.

\subsection{Archetype}

An archetype can be described as a formal expression of distinct, domain-level concepts which are reusable. Examples of such domain-level concepts include blood pressure, physical examination, or laboratory results which are usually defined by data constraints, and whose instances correspond to some reference information model [12]. An archetype is defined by a data structure, as well as its optionality and multiplicity. It is also defined by its data value constraints, and its significant bindings to natural language and terminology systems. It specifies the relationship that exists between data values within a data structure which is usually expressed in the form of algorithms, formulae or rules [8]. According to [8], an archetype consists of a formal and interoperable data specification as well as their relationships which are usually agreed upon within an electronic health record for documenting a particular clinical observation, evaluation, instruction or action. An archetype can also be described as a constraint domain entity model which expresses the configurations of data instances whose classes conform to an information model. An archetype is composed of three major parts [12]. These are the header section, the definition section and the ontology section. The header section is composed of a unique identifier for the archetype. The identifier is made up of a code which specifies the clinical concept defined by the archetype. Some descriptive information such as author, version and status are also described by the header section of the archetype. The definition section is composed of a constraint tree like structure which is developed from the information model. These structures limit the cardinality and content of the information model instances which conforms to the archetype [12]. An archetype instance is a knowledge artifact that defines how the EHR reference model hierarchy should be organized to represent the data for one clinical entry or care scenario. Archetype definitions are defined in a standardized form. They can be shared and used across record-sharing communities to define how locally-organized clinical data are mapped consistently regardless of the origin of the data [13]. Archetypes are the basic elements of communication between interoperating applications. This is because they define the minimum context that must be considered for safe communication. Thus, archetypes are agreed upon before communication. However, it is not feasible for all health professionals to agree on exactly all details of the archetypes associated to the data they would like to exchange [36].

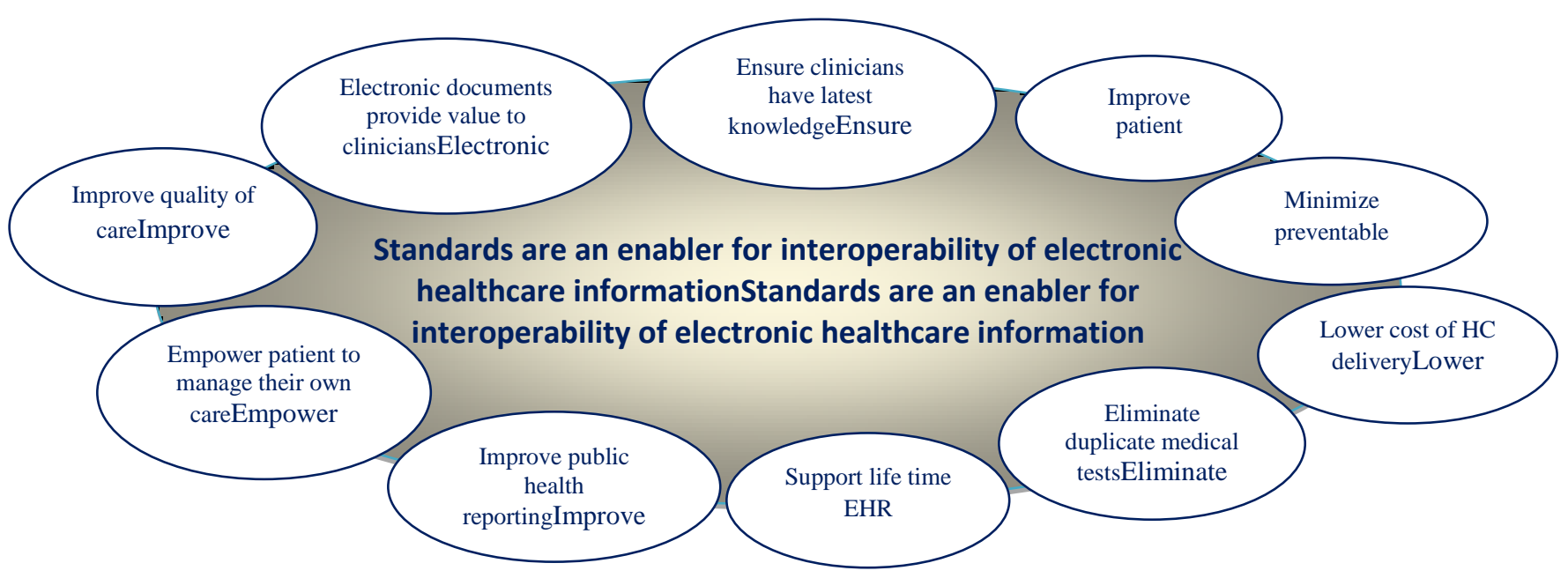

Fig 1: Benefits of Standards in Healthcare [11] 


\subsection{Web service and Service Oriented Architecture}

Web Services are modular, self-describing, and self-contained applications that are accessible over the internet [37]. Web services are considered to be a suitable solution for integrating disparate healthcare systems in a system heterogeneity environment. The basic technologies used by Web Services are XML, Simple Object Access Protocol (SOAP), Hyper Text Transfer Protocol (HTTP), Web Service Description Language (WSDL) and Universal Description, Discovery and Integration (UDDI). Web services implement a serviceoriented architecture. With open standards such as SOAP and XML, SOA supports interoperability in healthcare between services operating on disparate platforms as well as applications that are implemented in different programming languages. SOA plays the roles of a service provider and service consumer as described in figure 2. The service provider creates a web service and publishes its interface and access information to the service registry while the service consumer or web service client locates entries in the broker registry using various find operations and then binds to the service provider in order to invoke one of its web services. One of the disadvantages of using SOA for achieving semantic interoperability is the presence of data heterogeneity between interoperating services [14]. In addition, there is no consensus on a precise vocabulary and conceptual model for Web Services. Consequently, web services and service oriented architecture do not provide solutions for semantic interoperability in healthcare systems.

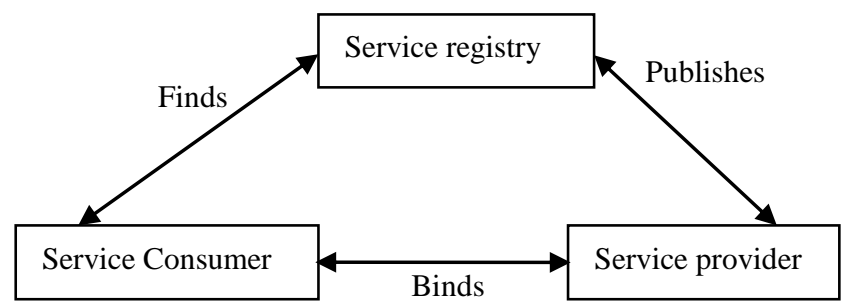

Figure 2: Service Oriented Architecture

\subsection{Clinical Terminologies}

A terminology is a compendium of words or phrases, otherwise known as terms, arranged in an orderly manner to represent the conceptual information of given knowledge domain of interest [15]. Clinical terminology according to [16] is defined as standardized terms and their synonyms which record patient findings, circumstances, events, and interventions with adequate specifications to support clinical care, decision support, outcomes research, and quality improvement; and can be sufficiently mapped to broader classifications for administrative, regulatory, oversight and fiscal requirements. Terminology is a vital tool for achieving semantic interoperability and consequently improving communication in healthcare, and ultimately benefiting patients [17]. The basic role of clinical terminology in healthcare is to provide a systematized and controlled vocabulary of clinical terms that can be used during data exchange. Common examples of clinical terminologies include International, Classification of Diseases (ICD) and Current Procedural Terminology (CPT). One of the drawbacks of clinical terminology is that terminologies can have several representations for the same clinical term (e.g. heart attack and myocardial infarction). In addition, the absence of a unified clinical terminology in the healthcare introduces semantic heterogeneity to the domain.

\subsection{Ontologies}

Ontology is a group of medical concepts in the context of healthcare, which are linked together hierarchically based on the underlying relationships among these concepts within the domain of interest [18]. Ontologies are composed of existing medical knowledge, through the systematic and explicit representation of medical definitions, concepts and processes, and provide a tool for constructing computer support systems that help clinicians to perform clinical tasks more effectively and efficiently. In Bio medical \& ontology Forums, ontology is described as a controlled vocabulary of well-defined terms along with their specified relationships, with the ability of being interpreted by both humans and computers [19]. In the context of computer and information sciences, ontology provides a vocabulary that describes a domain of interest and a specification of the meaning of terms used in the vocabulary [4]. They are generally employed to provide a uniform and standardized conceptualization of terms [20]. Ontologies are used to improve communication either between humans or computers by specifying the meaning of the concepts used in the communication process [21]. The essential components of ontologies are concepts, relationships and individuals or instances. The concepts are represented as entities that contain individuals, which meet the requirements' specification for the membership of the concepts. For instance, the concept Patient contains all the individuals which are patients in a given healthcare domain. Concepts are organized into superclass or sub-class hierarchy, which is also known as taxonomy. Individuals or instances are the objects the domain while relationships link two individuals together. Some of the pronounced benefits of ontologies in healthcare include:

a. Ontologies provide powerful and more interoperable information systems in healthcare by providing an explicit meaning of terms used by communicating systems.

b. Ontologies facilitates the transmission, re-use and sharing of patient and other health-related data

c. Ontologies facilitate the integration of knowledge and data across disparate systems in healthcare.

d. Ontologies also provide semantic-based criteria that support different statistical aggregations for different purposes in healthcare.

e. It improves the accuracy of diagnoses by providing real time correlations of symptoms, test results and individual medical histories through standards-based systems for systematic cross-checking diagnoses

f. Ontologies facilitate the development of semantic interoperability tools in healthcare

\section{ONTOLOGIES IN HEALTHCARE}

Ontologies in healthcare are developed to facilitate the reuse and exchange of medical data [18]. The basic advantage of using ontologies in the healthcare system is the ability to resolve semantic heterogeneity present within the data. Ontologies define the relationship between different types of semantic knowledge. They aid in the resolution of terms for queries and other general search strategies, thus improving the search results that are presented to clinicians [22]. Consequently, there are diverse ontologies in existence today 
in the healthcare domain. These ontologies are developed independently by healthcare excerpts in the healthcare domain. Some of the leading ontologies employed in healthcare include:

\subsection{The Medical Subject Headings}

The Medical Subject Headings (MeSH) thesaurus is a commonly used ontology in the healthcare domain. Mesh was produced by the National Library of Medicine for indexing, cataloguing, and searching for biomedical and health-related information and documents.

\subsection{Disease Ontology}

The Disease Ontology is a formal ontology of human disease developed at Northwestern University. The Disease Ontology is a community driven, open source ontology that is designed to show the relationship among disparate datasets through disease concepts. The mission the Disease Ontology (DO) is to provide open source ontology for the integration of biomedical data that is associated with human disease.

\subsection{Systemized Nomenclature of Medicine - Clinical Terms (SNOMED-CT)}

SNOMED-CT is developed by SNOMED International which is a division of the College of American Pathologists (CAP). It is owned, maintained, and distributed by the International Health Terminology Standards Development Organization (IHTSDO) [18]. It is an organized, comprehensive, computer processeable, multi-axial, and a controlled medical terminology. It is widely regarded as the most comprehensive clinical healthcare terminology in the world [23]. The major goal of SNOMED-CT is for indexing of medical records [23]. SNOMED-CT covers almost all clinical information such as diseases, findings, procedures, microorganisms and pharmaceuticals [24]. It is composed of a knowledge model which consists of elementary concepts, a set of attributes, as well as rules which states how individual elements are combined to form complex concepts [25]. It is made up of a set of more than 300,000 coded medical terms [26]. In SNOMED CT, logical terms such as "terms" and "concepts" are used to model different levels of entities. The concepts are arranged in an hypernymy or IS-A relationship hierarchy [26]. Concepts may have multiple parents; however, a parent cannot be its own child. Consequently, SNOMED-CT concept graph is acyclic. Concepts can also have roles. The concepts in SNOMED-CT are based on Descriptive Logics. Descriptions are terms or names (synonyms) assigned to a concept. SNOMED-CT is multilingual in nature. This is because SNOMED-CT is designed in both English and Spanish language concepts [17].

\subsection{NANDA (North American Nursing Diagnosis Association )Taxonomy II}

NANDA is the most extensively used classification of patient phenomena in the nursing domain. It is developed and maintained by NANDA International. NANDA taxonomy II has a hierarchical structure of 12 domains, 47 classes and 188 nursing diagnoses [15].

\subsection{Unified Medical Language System (UMLS)}

The UMLS was developed by the National Library of Medicine (NLM) for the purpose of solving two important obstacles to effective retrieval of machine-readable information which include the use of diverse names to express the same concept and the lack of a standard format for distributing terminologies [27]. The Unified Medical Language System (UMLS) is a collection of several controlled vocabularies in the healthcare domain. It allows these vocabularies to interact. UMLS can be viewed as a comprehensive thesaurus and ontology of biomedical concepts. The UMLS promotes interoperability in healthcare by incorporating and distributing key terminology, classification and coding standards, and associated resources [27]. Knowledge is organized by concept in UMLS. The UMLS graph is formed as a result of diverse relationship that exists among concepts. In addition, synonymous terms are also clustered to form a concept and concepts are linked to other concepts by means of various types of relationships, resulting in a rich graph. There are three major components of UMLS. These include the Metathesarus, the semantic network and the specialist lexicon [28]. The Metathesaurus is organized by concept, and each concept has specific attributes defining its meaning and is linked to the corresponding concept names in the various source vocabularies. Each concept in the Metathesaurus is assigned one or more semantic types (categories), which are linked with one another through semantic relationships. The scope of the Metathesaurus is determined by the scope of the source vocabularies, that is, the metathesarus represents different vocabularies that use different names for the same concept, or the same name for different concepts. The semantic network is a catalog of semantic types and relationships. UMLS has 135 semantic types and 54 relationships in total. The major semantic types are organisms, anatomical structures, biologic function, chemicals, events, physical objects, and concepts or ideas. The links among semantic types define the structure of the network and show important relationships between the groupings and concepts. The information about a semantic type includes an identifier, definition, examples and hierarchical information about the encompassing semantic types and associative relationships. Associative relationships within the semantic Network are very weak [29]. The specialist lexicon contains information about common English vocabulary and biomedical terms found in the UMLS Metathesaurus. Each entry in the lexicon contains syntactic, morphological and orthographic or spelling information.

\subsection{OpenGALEN}

OpenGALEN is an open-source project that is targeted at providing semantic integration in the Health care domain. It is a clinical terminology which is described in a formal and medically oriented language known as GRAIL (Galen Representation and Integration Language. GRAIL is a concept modeling language and a formal system for modeling medical concepts [24]. It was specially developed for specifying restrictions in the medical domains. OpenGALEN was developed for to be conveniently employed with different natural languages and integrated with different coding schemata. Medical terminologies are constructed in GALEN based on description logic models. The Common Reference Information Model is the most significant part of OpenGalen. This function of this model is to store clinical content such as medical concepts and it can also be used as a framework for specific knowledge about protocols or decision support. It is based on a semantically sound model of clinical terminology known as the GALEN COding REference (CORE) model [18]. The GALEN CORE top-level ontology establishes four general categories 18]:

i. Structure: This category is composed of abstract or physical things with parts that are time-independent (such as microorganism, protocol or heart). 
ii. Substances: These are continuous abstract or physical things that are time-independent, such as bile, drugs or radiation.

iii. Processes: These are changes that take place over time, such as irradiation, clinical act or breathing.

iv. Modifiers: These are used to improve or change the meaning of the other three categories, such as severe diabetes.

One basic drawback of ontologies in healthcare is that ontology remains unfamiliar to most healthcare providers [1]. The use of diverse ontologies in the healthcare domain raises heterogeneity problems to a higher level [2]. This heterogeneity could be in syntax, semantics, and terminological heterogeneity [4]

\section{ONTOLOGICAL HETEROGENEITY IN HEALTHCARE}

\subsection{Syntactic heterogeneity}

This occurs when two ontologies are not expressed in the same ontology language.

\subsection{Terminological heterogeneity}

This occurs when diverse clinical terms represent the same entities in different ontologies. This can be caused by the use of different natural languages. An example of this type of kind heterogeneity is heart and cardiac which are synonymous terms.

\subsection{Conceptual heterogeneity or semantic heterogeneity}

This is the collective term for different possibilities to model a domain. Semantic heterogeneity occurs whenever two contexts do not share the same interpretation of information (e.g. homonyms and synonyms). It can be divided into three sub problems. These include the difference in coverage, granularity and perspective. Coverage differences occur if ontologies are written from the same point of view, that is, they are written in the same context and with comparable vocabulary, but the part of the domain that is described differs and there are only overlapping parts. Difference in granularity occurs when the same section of the domain is described but the depth of details is not equal. If the point of view from which ontology is designed differs, there is a difference in perspective [33].

\subsection{Semiotic heterogeneity or pragmatic heterogeneity}

This is caused by the subjective interpretation of the used terms by humans. Terms can be considered regarding their context, such that terms with the same meanings are interpreted in different ways.

However, in order to resolve this ontological heterogeneity as well as achieve semantic interoperability in healthcare, ontologies have to be matched.

\section{A COMPARATIVE ANALYSIS OF EXISTING SOLUTIONS TO SEMANTIC INTEROPERABILITY IN THE HEALTHCARE DOMAIN}

\begin{tabular}{|c|c|c|}
\hline $\begin{array}{l}\text { Existing } \\
\text { Solutions }\end{array}$ & Advantages & Limitations \\
\hline Standardization & $\begin{array}{l}\text { The use of standards in } \\
\text { the healthcare domain } \\
\text { facilitates the } \\
\text { exchange of patient } \\
\text { and other health } \\
\text { related information } \\
\text { among heterogeneous } \\
\text { systems. }\end{array}$ & $\begin{array}{l}\text { Healthcare systems } \\
\text { do not adhere to the } \\
\text { same standard, this } \\
\text { results to severe } \\
\text { semantic } \\
\text { interoperability } \\
\text { problem in the } \\
\text { healthcare domain }\end{array}$ \\
\hline Archetype & $\begin{array}{l}\text { An archetype defines } \\
\text { the data structure, } \\
\text { data value constraints, } \\
\text { and the relationships } \\
\text { between data values } \\
\text { within a data structure }\end{array}$ & $\begin{array}{l}\text { There is no } \\
\text { possibility for all } \\
\text { healthcare providers } \\
\text { to agree on a } \\
\text { common archetype } \\
\text { during the exchange } \\
\text { of health-related } \\
\text { data }\end{array}$ \\
\hline $\begin{array}{l}\text { Web service } \\
\text { and service } \\
\text { oriented } \\
\text { architecture }\end{array}$ & $\begin{array}{l}\text { Web services and } \\
\text { SOA supports } \\
\text { syntactic } \\
\text { interoperability among } \\
\text { healthcare systems }\end{array}$ & $\begin{array}{l}\text { A major } \\
\text { disadvantage of web } \\
\text { services and SOA is } \\
\text { the existence of data } \\
\text { heterogeneity } \\
\text { between } \\
\text { interoperating } \\
\text { services. Also, there } \\
\text { is no consensus on a } \\
\text { precise vocabulary } \\
\text { and conceptual } \\
\text { model for Web } \\
\text { Services. }\end{array}$ \\
\hline $\begin{array}{l}\text { Clinical } \\
\text { Terminologies }\end{array}$ & $\begin{array}{l}\text { clinical terminologies } \\
\text { provide a systematized } \\
\text { and controlled } \\
\text { vocabulary of clinical } \\
\text { terms that can be used } \\
\text { during data exchange }\end{array}$ & $\begin{array}{l}\text { The lack of a unified } \\
\text { clinical terminology } \\
\text { in the healthcare } \\
\text { domain introduces } \\
\text { semantic } \\
\text { heterogeneity to the } \\
\text { domain. }\end{array}$ \\
\hline Ontologies & $\begin{array}{l}\text { Ontologies provide an } \\
\text { explicit representation } \\
\text { of medical definitions, } \\
\text { concepts and processes }\end{array}$ & $\begin{array}{l}\text { The use of diverse } \\
\text { ontologies in the } \\
\text { healthcare domain } \\
\text { introduces semantic } \\
\text { heterogeneity to the } \\
\text { healthcare system }\end{array}$ \\
\hline
\end{tabular}

\section{ONTOLOGY MATCHING}

Based on the diversity of ontologies in healthcare, there must be an establishment of semantic mappings among the ontologies in order to ensure interoperability. Ontology matching is the process of setting up conjunction between different ontologies without changing the original ontology, so that both sides can obtain a common understanding of the same object [31]. It can also be defined as a process of finding a corresponding entity with the same or the closest intended meaning between two or more ontologies [30]. Ontology mapping takes two ontologies as input and creates a semantic correspondence between the entities in the two input ontologies. [32] define ontology matching as thus: 
"Given two ontologies O1 and O2, matching one ontology onto another means that for each entity (concept $\mathrm{C}$, relation $\mathrm{R}$, or instance I) in ontology $\mathrm{O} 1$, we try to find a corresponding entity, which has the same intended meaning, in ontology $\mathrm{O} 2$ ".

Ontology matching can also be defined as the process where two ontologies with an overlapping content are related at the conceptual level and the source ontology instances are automatically transformed into the target ontology instances according to these relations [1]. Ontology matching is established after an analysis of the similarity according to certain metrics of the entities in the compared ontologies. The result of an ontology matching process is referred to as an alignement. An alignment is defined as a set of correspondences, which represent relations between different entities. A correspondence can be described by a 5-tuple

$<$ id, e, e', r, n>....

Where id is a unique identifier of the correspondence, $\mathrm{e}$ is an entity of the source ontology $\mathrm{O}, \mathrm{e}^{\prime}$ is an entity of the target $\mathrm{O}^{\prime}$, $\mathrm{r}$ denotes an alignment relation such as equivalence $(=)$, more general, overlapping and disjointness between the two entities and $\mathrm{n}$ gives a confidence value such as a similarity value.

\section{SIMILARITY MEASURES IN ONTOLOGY MATCHING}

Similarity measures form the basis of all matching algorithms, because it measures the degree of similarity between the ontologies to be matched. A formal notation for similarity measure is as given below [33]: A similarity function is defined as:

sim: $\mathrm{E} \times \mathrm{E} \rightarrow \mathrm{R}$

$\mathrm{E}=\mathrm{E} 1 \cup \mathrm{E} 2$.

$\mathrm{E}_{1}$ is the entity set of ontology $\mathrm{O}_{1}, \mathrm{E}_{2}$ the entity set of $\mathrm{O}_{2}$, which gets two entities as input and calculates a similarity value. [34] gave some examples of measures of similarity that can be used during ontology matching. These include:

\subsection{Terminological method}

This compares the labels of the entities/ concepts. It makes use of purely syntactic approaches as well as the use of lexicon such as Wordnet. The syntactic approach carries out the correspondence through measurements of dissimilarity of the chains while, the lexical approach computes the correspondence through the lexical relations such as synonymy, and hyponymy.

\subsection{Method of comparison of the internal structures}

This compares the internal structures of the concepts such as the interval of value, and cardinality of attributes.

\subsection{Method of comparison of the external structures}

This compares the relationships between entities and other components of the ontology. It involves the methods of comparison of the entities within the ontology and methods of comparison of the external structures by holding account of cycles.

\subsection{Semantic method}

This compares interpretations or the models of the entities in the ontology.

\section{THE CRITICAL NEEDS FOR ONTOLOGY MATCHING IN HEALTHCARE}

\subsection{Easy Interpretation of Medical Terms}

Healthcare professionals are required to have access to the detailed and complete health data as well as patient records across geographical boundaries in order to manage the safe and effective delivery of health services. These information are usually represented using diverse terminologies, which introduces semantic heterogeneity to the healthcare domain. This might prevent the easy interpretation of terms concerning a patient at the point of care. Consequently, clinical misinterpretation, knowledge mismanagement, misdiagnosis of the patient's illness or even death might arise. However, this can be avoided by identifying the correspondences between terms that are semantically related. This concept of ontology matching will enable the healthcare practitioners to identify similar terms that are represented heterogeneously, thereby facilitating the interpretation of medical terms.

\subsection{Reduction of Medical Errors}

The delivery of safe and effective health care is a challenge, particularly as the extent of medical errors is becoming apparent. The US Institute of Medicine reported that 100,000 US citizens die each year through medical errors [13]. This is because the healthcare domain lacks a unified terminology as well as a unified ontology, which leads to semantic heterogeneity and hence the problem of semantic interoperability. However, ontology matching helps to resolve ambiguities in ontological terms and concepts thereby aiding medical interpretation and thus reducing medical errors.

\subsection{Data Integration}

Ontology matching facilitates data integration in healthcare. For instance, the matching of an ontology for drug formulary for clinical pathways where a drug (a source entity) is matched onto a medication (target ontology), results in a transformed entity which captures the properties of the drug from the source ontology and the dosage information for a particular medical condition from the target ontology [35].

\subsection{Meaningful Exchange of Information}

Ontology matching facilitates the exchange of meaningful clinical information amongst healthcare institutes.

\subsection{Wider Support for Semantic Interoperability}

Ontology matching offers wider support for semantic interoperability. This is because it reconciles ontologies mismatches in different systems in healthcare.

\subsection{Information Re-use}

Ontology matching facilitates information re-use in healthcare.

\subsection{Guaranteed Patient safety}

With ontology matching, the quality of care will be increased, patient safety will be guaranteed, and the cost of healthcare will be reduced.

\section{CONCLUSION}

A major challenge facing the healthcare domain is the lack of semantic interoperability amongst healthcare systems. This is largely due to the fact that the healthcare lacks a unified terminological set. However, with the growing complexity of information and the increasing need to completely and 
correctly exchange information among different healthcare systems, the need for precise and unambiguous capture of the meaning of concepts is becoming apparent. Hence, the use of ontologies is to make explicit the meaning of terms used in healthcare systems in order to resolve semantic heterogeneity. However, the diversity of ontologies used in this domain raises semantic heterogeneity to a higher level which can be resolved by finding the matches or correspondences between the heterogeneous ontologies. This will result in semantic interoperability in healthcare.

\section{REFERENCES}

[1] Veli B., Gokce B. L., Asuman, D. and Yildiray., K. 2006. Artemis Message Exchange Framework: Semantic Interoperability of Exchanged Messages in the Healthcare Domain. Software Research and Development Center, Middle East Technical University (METU), Ankara Turkiye.

[2] Semantic Health Report 2009. Semantic Interoperability for Better Health and Safer Healthcare. European Commission, Informtion Society and Media.

[3] Stefan, S., Holger, S., Martin, B., and Barry S. 2009. Strengths and Limitations Of Formal Ontologies In The Biomedical Domain. Electronic Journal of Communication Information and Innovation in Health. pp. 31-45.

[4] Shvaiko, P. 2006. Iterative Schema-Based Semantic Matching. PhD Dissertation. International Doctorate School in Information and Communication Technology, University Of Trento.

[5] Trond, A. U., and Jochen, F. 2008. The Momentum of Open Standards - a Pragmatic Approach to Software Interoperability. European Journal of e-practice. Pp. 1-13

[6] Institute of Electrical And Electronics Engineers 1990. IEEE Standard Computer Dictionary: A Compilation of IEEE Standard Computer Glossaries. New York, NY.

[7] Gregory A.S., Kushel, R. B., and William, Y. 2009. Supporting Interoperability Using the Discrete-Event Modeling Ontology (Demo). Proceedings of The 2009 Winter Simulation Conference M. D. Rossetti, R. R. Hill, B. Johansson, A. Dunkin And R. G. Ingalls, Eds.

[8] Kalra,D. 2008. Getting The Right Level Of Semantic Interoperability For Now. Centre For Health Informatics and Multi-professional Education (CHIME). University College London.

[9] Beale, T., Heard M. D 2007. An Ontology-based Model of Clinical Information. MEINFO 2007, pp 760-766.

[10] Quinn, J. 2008. Health Information Technology Architecture vs. Semantic Interoperability. One Reason Why Health Information Technology (HIT) Interoperability Standards Can't Achieve the "Vision". Bellagio, Italy.

[11] Datta, G. (2010). HL7 International, Health Level Seven Introduction. Health Level 7 International, USA.

[12] Ozgur, K. and Asuman, D. (2006).Achieving Clinical Statement Interoperability using R-MIM and Archetypebased Semantic Transformations. European Commission and Scientific and Technical Research Council Turkey (TUBITAK).

[13] Semantic Health Report 2007. Semantic Interoperability Deployment and Research Roadmap. European Commission, Information Society and Media.

[14] Nagarajan, M., Verma, K., Amit P. S, John, M. and Jon., L. 2006. Semantic Interoperability of Web Services Challenges and Experiences. Department of Computer Science, University of Georgia, Athens GA, USA

[15] Hyeoun-Ae., P, and Nick, H. 2009. Clinical Terminologies: A Solution for Semantic Interoperability. Journal of Korean Society of Medical Informatics Vol. 15, No 1., pp 1-11.

[16] Chute., C.G. 2000. Clinical classification and terminology: Some history and current observations. Journal of Am Medical Information. vol. 7. Pp. 298303.

[17] Ryan, A. 2007. Towards Semantic Interoperability in Healthcare: Ontology Mapping from SNOMED-CT to HL7 version 3. School of Economics and Information Systems. The University of Wollongong, Northfields Avenue, Wollongong.

[18] Waraporn, P., Meesad, P. and Clayton G. 2010. Proposed Ontology Based Knowledge and Integration Framework. IJCSNS International Journal of Computer Science and Network Security, Vol.10 No.3, pp. 30-36.

[19] Kenjige., P. 2010. Usage of Medical Ontology In EA. PK Technologies.

[20] Costa, A., Renata, S. S., Guizzardi, G., and Jos'e, C. 2004. COReS: Context-Aware, Ontology-Based Recommender System for Service Recommendation. Department of Computer Science, UFES, Vitoria-ES, Brazil, ITC-irst, Trento-Povo, Italy and Laboratory of Applied Ontologies (ISTC-CNR), Trento, Italy.

[21] Bittner, T.,Donnelly, M and Winter, S.. 2006. Ontology and Semantic Interoperability. Institute for Formal Ontology and Medical Information Science (IFOMIS) Saarland University.

[22] Ashiq, A., Peter, B., Andrew, B., Tamás, H., Richard, M., Kamran, M., Dmitry, R. and Jetendr, S. 2007. The Requirements for Ontologies in Medical Data Integration: A Case Study. CCS Research Centre, CEMS Faculty, University of the West of England, Coldharbour Lane, Frenchay, Bristol, UK.

[23] Fabozzi, N. 2010. Kaiser's Donation of its Convergent Medical Terminology Dictionary Puts the Spotlight on the Role of Clinical Terminology Services in Driving Meaningful Use of EHRs. Healthcare and Life Sciences, Frost and Sullivan.

[24] Alan, R. 2001. Description Logics in Medical Informatics. School of Computer Science, University of Manchester, England.

[25] Fang, C., Norm, A., and Skip, P. 2009. An Agent-based Knowledge Management Framework for Electronic Health Record Interoperability. Journal Of Emerging Technologies In Web Intelligence, Vol. 1, No. 2, pp. 119-127.

[26] Calin Cenan, Gheorghe Sebestyen, Gavril Saplacan, Dan Radulescu. Ontology-Based Distributed Health Record 
Management System. Dept. of Computer Science, Technical University of Cluj Napoca.

[27] Bodenreider, O., Smith, B., Kumar, A. and Burgun, A. (2004) Investigating Subsumption In DL-Based Terminologies: A Case Study in SNOMED-CT. First International Workshop on Formal Biomedical Knowledge Representation, Pp. 12-20.

[28] Bodenreider, O. 2004. The Unified Medical Language System (UMLS): integrating biomedical terminology. Nucleic Acids Research Journal. Vol. 32, No 1; SUPP, pp 267-270.

[29] UMLS 2007. Unified Medical Language System. Bethseda, MD: National Library of Medicine.

[30] Interop 2008. State of the Art Report. Ontology Interoperability. Information Society Technologies.

[31] Rung-Ching, C., Bo-Ying, L., and Cho-Tscan, B. 2009. Using Domain Ontology Mapping for Drugs Recommendation. Department of Information Management, Chaoyang University Of Technology, Taiwan.

[32] Ehrig, M, and Staab, S. 2004. QOM - Quick Ontology Mapping. International Semantic Web Conference. Vol. 3298, pages 683-697.
[33] Katrin, S. Z. 2010. Instance-Based Ontology Matching and the Evaluation of Matching Systems. InauguralDissertation. Department of Computer Science, Heinrich Heine University of Dusseldorf, Germany.

[34] Rahm, E., and Bernstein, P. 2001. A Survey of Approaches to Automatic Schema Matching. VLDB Journal. Pp 334 - 350 .

[35] Puri, C., Gomadam, K., Jain, P., Yeh, P., and Verma, K. 2011. Multiple Ontologies in Healthcare Information Technology: Motivations and recommendations for Ontology Mapping and Alignments. International Journal of Biomedical Ontologies, Buffalo, New York, USA.

[36] Damon, B. and Jesus, B. (2009). Archetype Alignment: A Two-Level Driven Semantic Matching Approach to Interoperability in the Clinical Domain. TeaPOT: People Oriented Technology Conference Papers, Dublin Institute of Technology.

[37] Wei, C., Doong, S., And Sung, S. (2007). On Resolving The Problem Of Semantic Heterogeneity In Clinical Information Systems. Department of Computer Information Systems BMCC, City University of New York, New York, USA. 